\title{
Thermal Analysis of Magnetic Polymer Nanospheres for Drug Targeting
}

\author{
A. Juríková, K. Csach, J. Miškuf, M. Koneracká, V. ZÁVišová \\ AND P. KOPČANSKÝ
}

Institute of Experimental Physics, Slovak Academy of Sciences, Watsonova 47, 04001 Košice, Slovakia

Poly(D,L-lactic-co-glycolic) acid (PLGA) polymer nanospheres loaded with different input amounts of anticancer drug taxol were prepared by the modified nanoprecipitation method. Magnetite was incorporated into the polymer nanospheres to impart them superparamagnetic properties. Thermal properties of the drug loaded magnetic polymer nanospheres were characterized using differential scanning calorimetry and thermogravimetric analysis. The solid state solubility of taxol in PLGA nanospheres and the influence of external magnetic field on their thermal stability were estimated. The investigations have revealed that the samples of dried taxol loaded magnetic PLGA nanospheres undergo mass loss at three stages during heating.

PACS numbers: 47.65.Cb, 65.80.-g, 47.63.mh

\section{Introduction}

Biodegradable nanospheres formed from PLGA polymers have been extensively investigated for various drug delivery applications [1, 2]. Magnetic drug targeting is one of the various possibilities of drug targeting. This technique is based on binding a selected anticancer drug with magnetic fluids into a pharmacologically stable formulation. Magnetic polymer nanospheres (NPs), prepared from organic and inorganic components, have unique characteristics due to the specific properties of the blend. The constituents of magnetic polymer nanospheres play different roles: the polymer matrix acts as a shell, reservoir, and vehicle for the active component, whereas magnetite is the component that makes targeting possible by external magnetic field manipulation.

Thermoanalytical methods (differential scanning calorimetry (DSC) and thermogravimetric analysis (TGA)) are very useful tools in the characterization of the NPs and interactions in polymer-drug systems $[2,3]$. The aim of the work was to prepare taxol loaded magnetic polymer NPs, to characterize their thermal properties and to estimate the solid state solubility of the drug and the influence of external magnetic field on the thermal stability of the NPs.

\section{Experimental}

In the experiment, PLGA polymer with D,L-lactide to glycolide ratio of 85:15 was used due to its biodegradable nature and biocompactibility with cells and tissue. Taxol (TAX), an important anticancer drug, was chosen for encapsulation to the polymer because of its significant role against a wide range of tumours. Magnetite $\left(\mathrm{Fe}_{3} \mathrm{O}_{4}\right)$ was prepared by co-precipitation of ferric and ferrous salts in an alkali aqueous medium [4]. To prepare stable colloidal suspension of magnetic particles, sodium oleate as a first surfactant was used to prevent their agglomeration. Poly(ethylene glycol) (PEG) as a second surfactant was added to the system magnetite-sodium oleate to improve biocompatibility of magnetic material [4].

The modified nanoprecipitation method was used for entrapment of magnetic fluid and anticancer drug into polymer nanospheres [4]. After the sphere formulation, the thermal behaviour of the prepared NPs was characterized by DSC and TGA methods. DSC measurements were performed using Perkin Elmer DSC 7 calorimeter at the rate of $10^{\circ} \mathrm{C} / \mathrm{min}$ up to $250{ }^{\circ} \mathrm{C}$ in nitrogen atmosphere. The thermogravimetric investigations were carried out using a SETARAM thermobalance model TGDTA92 at the heating rate of $10^{\circ} \mathrm{C} / \mathrm{min}$ up to $900^{\circ} \mathrm{C}$ in Ar atmosphere.

\section{Results and discussions}

Magnetic PLGA NPs loaded with different input amounts of taxol and with the mean diameter of 200-250 nm were prepared [4]. Figure 1 shows the relationship between TAX loading and the enthalpy change of TAX melting. In the inset there are DSC traces of the dried magnetic PLGA NPs loaded with TAX amount varying up to $37.5 \mathrm{wt} \%$. In DSC traces, endothermic peak (about $55^{\circ} \mathrm{C}$ ) refers to the glass transition temperature of PLGA. At the melting temperature of taxol, the observed endothermic process (peak II below $200^{\circ} \mathrm{C}$ ) corresponds to the melting of the residual undissolved drug. Endothermic peak III (about $120^{\circ} \mathrm{C}$ ) can be associated with the presence of surfactants in the used magnetic fluid encapsulated to polymer NPs. 


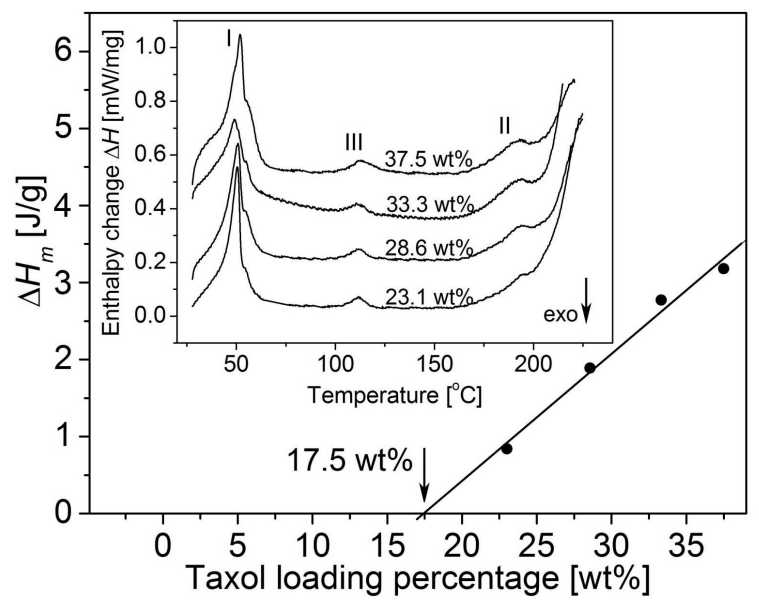

Fig. 1. The relationship between the taxol loading and the enthalpy of taxol melting. The inset shows DSC traces of PLGA NPs with indicated input taxol loading.

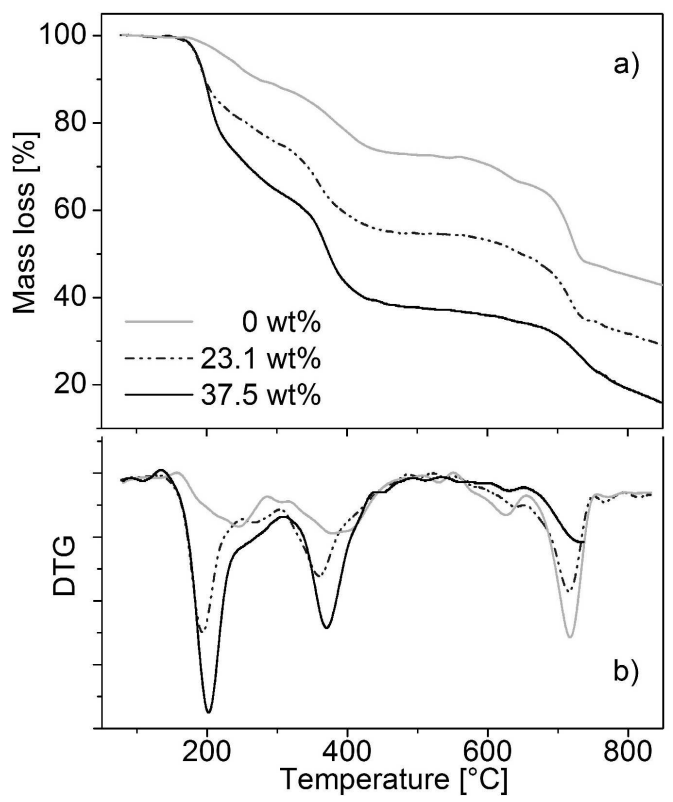

Fig. 2. Temperature dependence of the mass loss (a) and its derivative (b) during heating of magnetite PLGA NPs with indicated taxol loading.

To determine the drug solid-state solubility in the polymer, the values of the enthalpy changes at melting temperatures of the free drug $\Delta H_{\mathrm{m}}$ were considered. These values were plotted as a function of TAX loading percentage $m_{\mathrm{TAX}} /\left(m_{\mathrm{TAX}}+m_{\mathrm{PLGA}}\right)$. X-axis intercept provided by linear regression of the data gives a result of $17.5 \mathrm{wt} \%$, that means, when the drug was bound by the NPs, it was possible to bind a maximal amount of about $21.2 \mathrm{mg}$ of taxol into $100 \mathrm{mg}$ of the used polymer without excess observed by DSC.

Figure 2 shows the thermal decomposition of the dried magnetite NPs with variable taxol loading. The samples

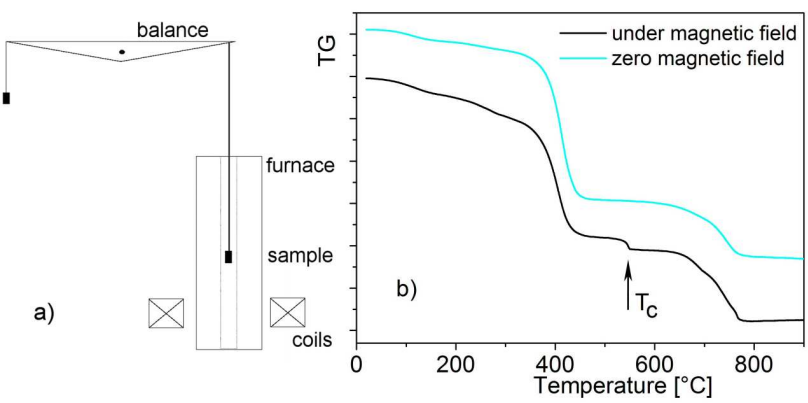

Fig. 3. (a) A schematic sketch of the modified TG apparatus. (b) The weight changes of pure magnetic NPs at linear heating under and without external magnetic field.

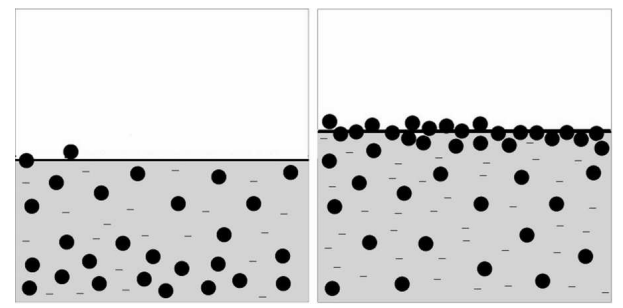

Fig. 4. Schematic representation for an explanation of differences in evaporation of PEG during heating under magnetic field (left) and without magnetic field (right).

lose their mass at three stages during heating, they undergo the melting or decomposition and evaporation of components. The mass loss corresponds to the amount of evaporated components. First, the molted PEG and TAX evaporate above $200^{\circ} \mathrm{C}$, sodium oleate above $360^{\circ} \mathrm{C}$ and the evaporation of PLGA causes mass loss at temperatures above $700^{\circ} \mathrm{C}$. The TG residue at $850{ }^{\circ} \mathrm{C}$ for pure magnetic NPs was $42.8 \mathrm{wt} \%$ and for taxol loaded magnetic NPs up to $15.9 \mathrm{wt} \%$. The $26.9 \mathrm{wt} \%$ difference between up and down thermograms was associated with the taxol presence. Derivative TG curves in Fig. 2b show the uniformity of the decomposition of the nanospheres.

Modified experimental apparatus for thermogravimetric measurements under external magnetic field can be seen in Fig. 3a. The weight changes of pure magnetic NPs during linear heating under external magnetic field and without it are shown in Fig. 3b. The arrow indicates the Curie temperature $T_{\mathrm{c}}$ and it was found to be about $546^{\circ} \mathrm{C}$. The higher evaporation of PEG under magnetic field gradient can be caused by the mixing of melt by moving solid magnetic particles. Another mechanism can be associated with increasing the concentration of magnetic particles in areas near the free surface of melted polymer NPs without external magnetic field. Schematic representation for a possible explanation of differences in evaporation of PEG at heating under and without magnetic field is displayed in Fig. 4. 


\section{Conclusion}

Magnetic PLGA nanospheres with variable loadings of taxol were succesfully prepared and thermally characterized. DSC measurements gave an estimation of taxol loading capacity at $21.2 \mathrm{mg}$ of taxol into $100 \mathrm{mg}$ of PLGA. The results confirmed incorporation of the drug taxol in the PLGA polymer nanospheres. The stepwise mass decrease upon the heating was connected with the decomposition of the prepared nanospheres. The investigations revealed that the samples lose their mass at three stages during heating. Two different mechanisms were used for the explanation of higher evaporation of PEG under external magnetic field.

\section{Acknowledgments}

This work was supported by implementation of the project No. 26220120021 provided by the European Re- gional Development Fund. The authors are also grateful to the Grant Agency VEGA and the Centre of Excellence "Nanofluid" of Slovak Academy of Sciences.

\section{References}

[1] Q.A. Pankhurst, J. Connolly, S.K. Jones, J. Dobson, J. Phys. D, Appl. Phys. 36, R167 (2003).

[2] J. Panyam, D. Williams, A. Dash, D. Leslie-Pelecky, V. Labhasetwar, J. Pharm. Sci. 93, 1804 (2004).

[3] C. Dubernet, Thermochim. Acta 248, 259 (1994).

[4] M. Koneracká, M. Múčková, V. Závišová, N. Tomašovičová, P. Kopčanský, M. Timko, A. Juríková, K. Csach, V. Kavečanský, G. Lancz, J. Phys., Condens. Matter 20, 204151 (2008). 